Cytogenet Genome Res 1997;77:319-320

\title{
Subject Index, Vol. 77, 1997
}

This index does not contain items of Vol.77, no. 1-2 (Abstracts of the 1st European Cytogenetics Conference) and Vol. 77, supplement 1 (Abstracts of the International Conference on

Chromosome 21 and Medical Research on Down Syndrome).

Animals

Ateies paniscus chamek (spider

monkey) 314

Babyrousa babyrousa (babirusa) 273

Bos indicus (zebu) 238

Bos taunts (cattle) 223, 238

Chinese hamster 228

Felis cattus (domestic cat) 211

Macropus eugenii (tammar wallaby)

242

mouse 180, 264, 285

Muntiacus muntjac vaginalis (Indian

muntjac) 223

Muntiacus reevesi (Chinese muntjac)

223

Neotragus moschatus (suni antelope)

218

rat 266

Sorex araneus L. (shrew) 201

Taurotragus oi $\gamma \mathrm{x}$ (eland antelope) 218

Tayassu pecari (white lipped peccary)

273

Banded chromosome analysis antelope(G, C)218 babirusa(Ag, GTG) 273 bovine(GTG) 238

bovine(R) 306 cat(G, DAPI)211 Chinese hamster(G) 228 human(DAPI) 167, 175, 178, 207, 232, 252,253,257,259,268,278,285,

288,296 human(DAPIG) 176 human(G) 169 human(GTG) 190, 192 human(R) 185,308

mouse(D $\Lambda$ PI) 285 mouse(R) 304 muntjac(DAPI) $223 \operatorname{rat(DAPI)~} 266$ white lipped peccary(Ag,

GTG) 273

Chromosome aberration

deletions 246, 296

fusions 223

isodisomy 296

pericentric inversions 238

rearrangements 218 Comparative gene mapping

antelope 218

bovine Y 238 
cat 211

muntjac 223

shrew 201 spider monkey 314 tammar wallaby 242 wild pig 273

Evolution

ancestral chromosomes 211,218, 242 antelope X chromosome 218 human chromosome 10242

marsupial chromsome 1242 muntjac karyotype 223 pig rDNA 273

spider monkey chromosome 3314 tandem gene duplication 207,

Flow cytometry

cat chromosome probes 211

human chromsomes 211 Fluorescent in situ hybridization (FISH)

$6 \mathrm{p}$ telomer specific 175

breakpoint determination 232

chromosome region specific painting probes 192,238

comparative chromosome painting 218, 223,238,242

comparative genomic hybridization 253,296

deletion analysis 246

direct $\mathrm{R}$ banding 185,261

G-banding after FISH 228

interphase FISH 246

multicolor FISH 175, 192, 246, 259

reciprocal chromosome painting 211

restriction endonuclease digestion 228

reverse FISH 232

Gene mapping babirusa

RNR1, RNR2, RNR3 273 bovine

NGFB 306 Chinese hamster

TP53 human

64 expressed sequence-tagged

sites (ESTS) 197

AAC1, AAC2, AACP290

ACACB 176

ATP5A, ATP5AP1 253

C1QBP283

CMOAT 285

COL3A1 259

COX6Al, COX6AlP167

CYRN2 178

DAP3 252

IL12RB1, IL12RB2 257

MCM4 268

MNBH 182

NEB 259

PTK6 271

RAB7L1 261

RPL32 190

SATB1 205 
STAT1, STAT4 207

WNT10B

WNT7B 288

ZNF188 185 mouse

Cnioat 285

Hmoxl, Hmox 2 ISO

SicI7a2 304

üfdll 264 rat

Hkl, Hk2, Hk3, Gck 266 shrew

ПDH2, PP, GPT, ADK, GUSB,

ACP2, AK1, AK3, GOT2, PEPD,

GH, PGM2, ENO1, OTC, ACY1,

GOT1 201 white lipped peccary

RNR1, RNR2, RNR3 273 Genetic damage

chromosome gain and loss 296 gene amplification 253

Heterochromatin

azacytidine induced decondensation

178 "

under methylation 308 Human disorders

bladder cancer 290

breast cancer 290

cri-du-chat syndrome 246

DiGeorge syndrome 264

Down syndrome 182

Dubin-Johnson syndrome 285

Ehlers-Danlos syndrome 259

hypophosphatemia 304

ICF syndrome 308

immunodeficiency 257

mental retardation 182

neurodegenerative diseases 205

retinoblastoma 253

skin disorders (epidermolysis bulbosa

simplex, hyperkeratosis, palmoplantar

keratoderma) 169

Velocardialfacialsyndrome 264

Wilms tumor 296

\section{KAKGEK}

E-Mail karger@karger.ch Fax+ 41613061234 http://www. karger. ch

(C) 1997S. Karger AG, Basel

319

Hybrids

chromosome 8 hybrid panel 197 human-rodent panel 278, 283 human-hamster 232 radiation hybrid 232 shrew-rodent 201 somatic cell hybrid panel 167

Karyotype 
suni antelope 218 white lipped peccary 273

Linkage

$2 q$ fragile site 259 AAC loci on 8p22 290 keratin gene locus on 17q 169 MCM4 and PRKDC 268 shrew genes 201

Microdissection and microcloning

abnormally banded regions (ABRs)

192

bovine Y 238

Physical mapping 5p deletions 24664 ESTS on chromosome 8197 6p specific telomers 175

8 p22 290

chromosome 5 hybrid panel 232 cri-du-chat critical region 246 type I keratin gene locus 169

Repeats

Alu sequence methylation 308 Ribosomal RNA gene (rDNA)

babirusa and peccary 273

Restriction mapping

AAC loci on 8p22 290 chromosome 5 mapping panel 232 cytokeratin type 1 genes 169

Sequence

Chinese hamster TP52 228

ESTR38268 182

MNBH182

RAB7L1 261

WNT10B 278

ZNF188 185 Sex chromosomes

Bovine Y 238

Techniques

direct selection of chromosome region-specific cDNAs 192 CGH screening of solid tumors 296

320

Cytogenet Cell Genet Vol. 77, 1997 